ISBN 978-81-933894-1-6

5th International Conference on Chemical, Agricultural, Biological and Environmental Sciences

(CAFES-17)

Kyoto (Japan) April 18-19, 2017

\title{
Catalytic degradation Methylene blue dye by green synthesized silver nanoparticles
}

\author{
Himadri Sekhar Samanta ${ }^{1}$ and Chiranjib Bhattacharjee ${ }^{1}$ \\ ${ }^{1}$ Department of Chemical Engineering, Jadavpur University, Kolkata - 700032 \\ India.
}

\begin{abstract}
In this study silver nano-particles were successfully synthesized by using Eucalyptus leaf extract. The aqueous leaf extract was added to silver nitrate solution and after incubation, pale yellow colour of reaction mixture was changed to brownish colour which indicates the reduction of silver ions to silver nanoparticles.The AgNPs were characterized by Ultraviolet-visible (UV-vis) spectrometer, FTIR, and Transmission electron microscopy (TEM). The XRD confirmed the crystalline nature and TEM showed spherical AgNPs. The degradation of methylene blue was carried out in the presence of silver nanoparticles in the visible region, using as a promising catalytic agent. The absorption spectrum showed the decreased peaks for methylene blue at different time intervals. Initially absorption peaks at $660 \mathrm{~nm}$ for Methylene blue dye were decreased gradually with the increase of exposure time.
\end{abstract}

Keywords: Green Synthesis, Eucalyptus leaf extract, Silver nanoparticles, dye degradation.

\section{Introduction}

It is well known that nano-biotechnology and nano-toxicology are the most dynamic inter disciplinary areas of research in contemporary material science where by plants and other natural recourses have a significant role in the synthesis of biogenic coated advanced nanoparticles. Nanomaterials have been receiving attention due to their unique physical and chemical properties compared with their larger-size counterparts[1]-[3]. The noble metal nanoparticles have been fabricated by chemical reduction with stabilising reagents (NaBH4, citrate, or ascorbate) [4], thermal decomposition [5], and photo reduction in reverse micelles [6] etc. Many of these approaches are expensive, consume a lot of energy, result in low yields, and the chemicals used in their production are toxic and hazardous [7-8]. Currently green synthesis of metallic nanoparticles is an emerging science in the field of nanotechnology and nanomedicine. Due to its wide applicability in various fields, "green chemistry" has been evolved to a widespread scope. Traditionally, chemical reduction has been the most groundwork for synthesis of metallic nanoparticles which often involves use of toxic chemicals are utilized to involve in most of the synthetic protocols.In order to avoid the use of toxic chemicals, organic solvents, several researchers attempt to develop the metallic nanoparticles by environmental friendly and biological approaches. Green approach of nanoparticles synthesis by biological entities has been gaining great advantages which are environmental benign, less toxic, and time consuming; and also it is a single step process.

Currently, plant and plant derived materials are used for nanoparticles synthesis which is more compatible than the microbe-mediated nanoparticles synthesis process because they eliminate the culture maintenance and are easy to handle. Silver nanoparticles have also been fabricated using plant extracts as reducing and capping agents. The main advantages of using plant extracts are that the process is simple, cheap, scaling-up, ecofriendly, and safe [9-12].Eucalyptus leaves (ELs) contain a wide range of active components and the research is still on the hunt for their potential uses as they are rich in polyphenols, including flavonoids and tannic acids, 
organic acids and volatile oils. In this study, a novel approach for synthesis of Ag-NPs was developed using ELs extract, which is a simple, economical and environmentally benign method. Methylene Blue(MB) is traditionally used as a dye for materials such as silk, leather, and paper. It is highly toxic to mammalian cells, carcinogenic and can cause skin irritation. Therefore, removal of MB from effluent is essential to protect the environment. Conventional biological treatment in removing dyes from wastewaters is generally ineffective as the dyes are resistant to microorganisms. Moreover, the physico-chemical treatment methods are ineffective at higher effluent concentrations. In recent years, Nanotechnology has been extended to the wastewater treatments. Due to high surface area AgNPs exhibits an enhanced reactivity. To the best of our knowledge till date there is no study available on catalytic activity of AgNPs in reduction of MB dye using leaf extract of Eucalyptus leaf extract. Moreover, the catalytic degradation of synthetic dye Malachite green by aqueous leaf extract in presence of AgNPs was investigated.

\section{Experimental}

\subsection{Materials and Chemicals}

In this study, Eucalyptus citriodora (EC) are used as the biomass materials. These EL was collected on the campus of Jadavpur University in Jadavpur, India. After being washed thoroughly, EC was dried in 40 C, then smashed finely and the powder screened through 90-mesh sieve, finally stored for late use. Silver nitrate (AgNO3) was used as the source of Ag ions, required for the synthesis of Ag-NPs. Deionized water is used in all experiments.

\subsection{Synthesis of Ag-NPs}

$6 \mathrm{~g}$ EL powder was bathed with $150 \mathrm{ml}$ deionized water at $50 \mathrm{C}$ for $1 \mathrm{~h}$, after that, the extract was filtered and stored at $4 \mathrm{C}$ for further experiments. In a typical reaction procedure, $50 \mathrm{ml}$ of EL extract was added to 25 $\mathrm{ml} \mathrm{5m} \mathrm{M}$ aqueous AgNO3 solution, with stirring at $30 \mathrm{C}$. The color of the solution changed immediately from light yellow to reddish brownafter the extract was added. The obtained Ag-NPs were centrifuged at 10,000 rpm for $10 \mathrm{~min}$, subsequently washed three times and dispersed in deionized water to eliminate the non-coordinated materials.

\subsection{Catalytic Performance Test}

Methylene blue purchased from $\mathrm{CDH}$ chemicals, India was used for this study. In order to assess the catalytic activity of synthesized AgNPs, two reactions were carried out and absorbance values were monitored using UV-visible spectrophotometer. The values of absorption maxima (max) at $664 \mathrm{~nm}$ were compared, with that of methylene blue. The schematic representations of the two reactions are depicted in Fig. 1.

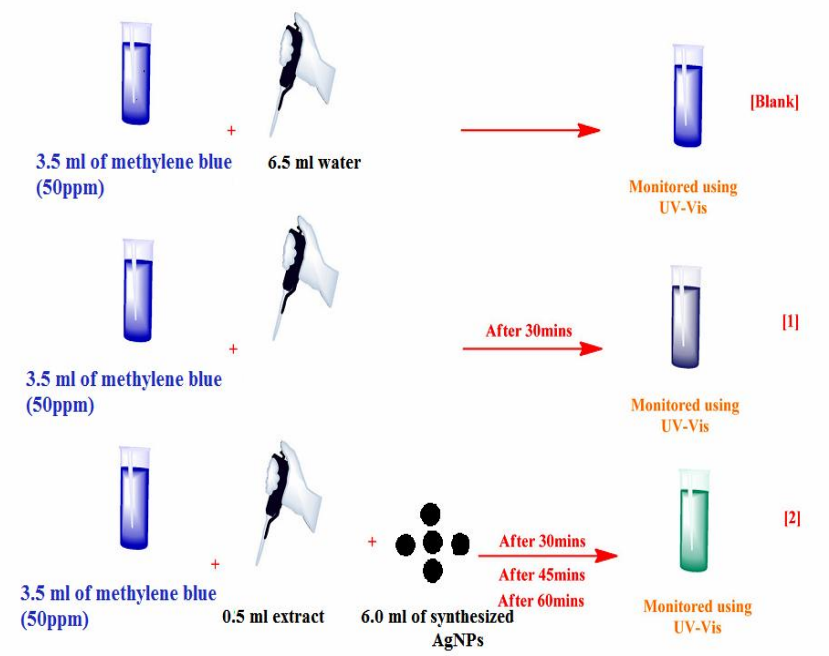

Fig. 1 Schematic representations of the reactions for evaluation of the effect of synthesized AgNPs on the reduction of methylene blue 


\section{Result and Discussion}

The formation of Ag-NPs is relevant to the coherent oscillation of SPR at the surface of NPs [13] and scanned with a UV-vis spectrometer (Varian Cary 50Bio) in the wave-length region between 300 and $600 \mathrm{~nm}$. Fig. 2 shows the UV-vis absorption spectra of the AgNPs synthetized with leaf extract obtained from EU shows peaks at at $440 \mathrm{~nm}$.

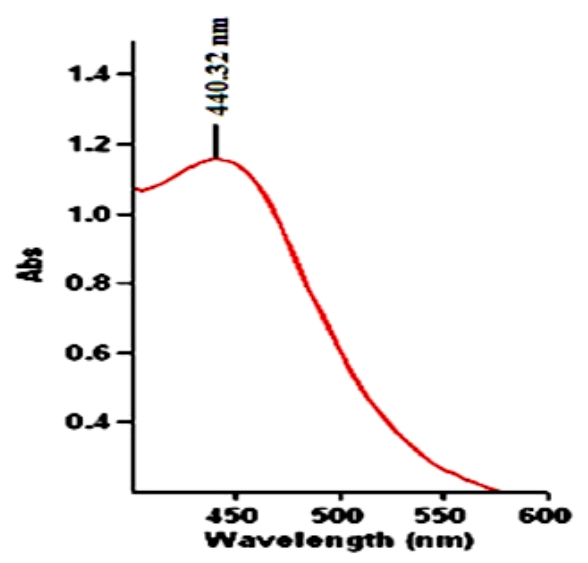

Fig.2 UV-visible absorption spectra of synthesized AgNPs.

Meanwhile, XRD of dry Ag-NPs powder was measured on an XRD (D/MAX-2500 V) operated under the condition of $40 \mathrm{kV}$ and $50 \mathrm{~mA}$ with CuK $\alpha$ radiation $\left(\lambda^{1 / 4} / 40.1542 \mathrm{~nm}\right)$. The XRD patterns (Fig. 3) analysis of NPs indicate that synthesized Ag-NPs are crystalline and the four distinct diffraction peaks at 38.111, 44.301, 64.441 and 77.401 which could be assigned to (1 11 1), (2 $\left.\begin{array}{lll}2 & 0\end{array}\right),\left(\begin{array}{lll}2 & 2 & 0\end{array}\right)$ and (3 111$)$ planes of face-centered cubic Ag-NPs respectively are in agreement with the database of Joint Committee on Powder Diffraction Standards (JCPDS No. 99-0094).

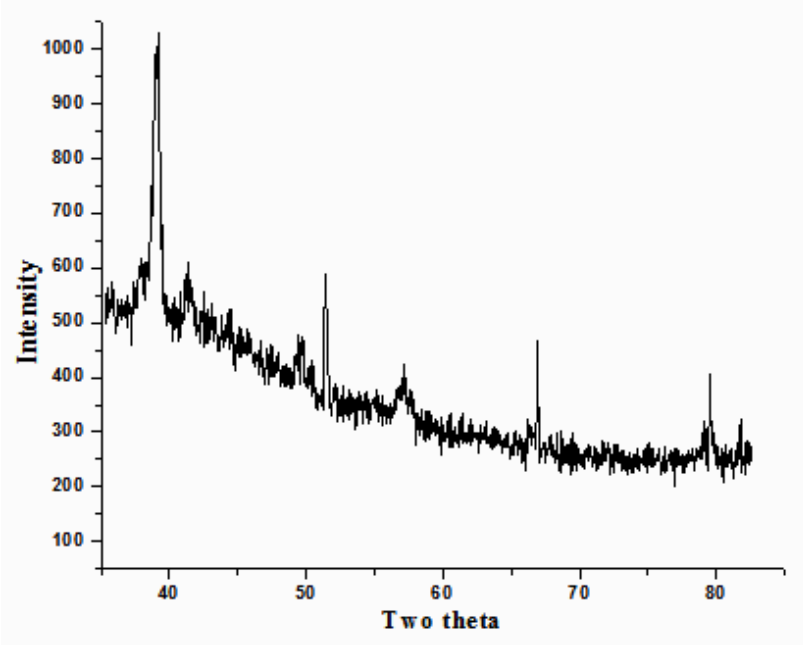

Fig. 3 XRD patterns of synthesized AgNPs.

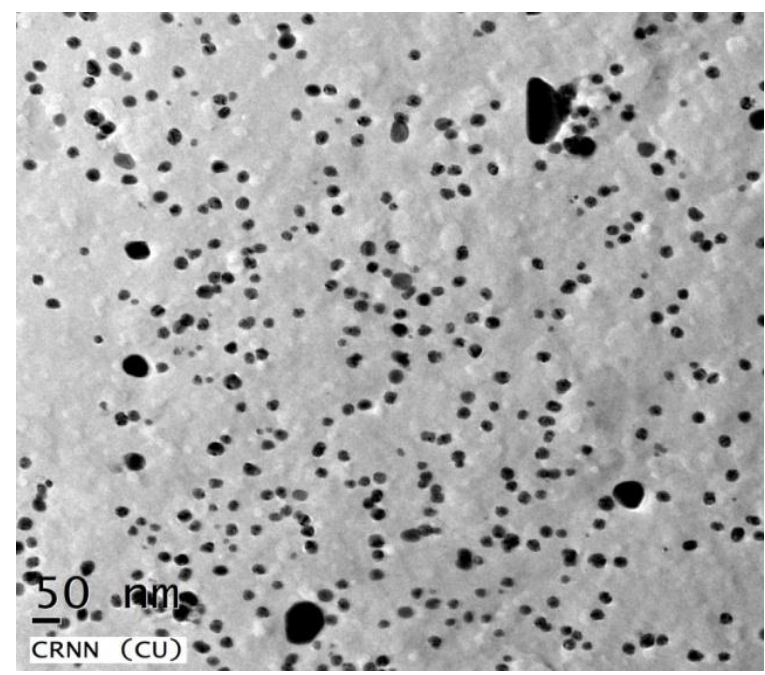

Fig. 4 HR-TEM images of synthesized AgNPs

The structure and morphology of the green synthesized Ag-NPs using the extract of EU were further confirmed by the HR-TEM (JEOL JEM 2100 High Resolution Transmission Electron Microscope operating in the range of $200 \mathrm{kV}$ ) micrograph images, showing at the scales of 100 and $10 \mathrm{~nm}$ (Fig. 4). The images depict 
that the Ag-NPs are spherical in shape, with the particles size distribution between 10 to $40 \mathrm{~nm}$ and an average diameter of $25 \mathrm{~nm}$, which is in fairly good consistency of the average crystallite size calculated from XRD analysis.The present study tried to use the FTIR spectra (Perkin Elnmer spectrum 2), presented in Fig. 3 to identify the possible reducing and stabilizing biomolecules involved in the NPs synthesis. Shown in Fig. 5B, the bands at $2925.44(\mathrm{C}-\mathrm{H}$ stretching vibration) and $1043.56 \mathrm{~cm} 1$ (C-O stretching) were observed, which are typical for $\mathrm{O}-\mathrm{H}$ stretching of alcohols or phenols, and the band at $1623.24 \mathrm{~cm} 1$ is characteristic of aromatic $\mathrm{C}=\mathrm{C}[7,18]$, respectively. After NPs synthesis in Fig. 5A, the bands at 2925.44, 1623.24 and $1043.56 \mathrm{~cm}$ 1shifted to $2929.28,1633.08$ and $1054.49 \mathrm{~cm} \mathrm{1,} \mathrm{respectively,} \mathrm{which} \mathrm{could} \mathrm{be} \mathrm{assumed} \mathrm{that} \mathrm{the} \mathrm{phenolic}$ hydroxyl groups are involved in the synthesis as reducing agents.

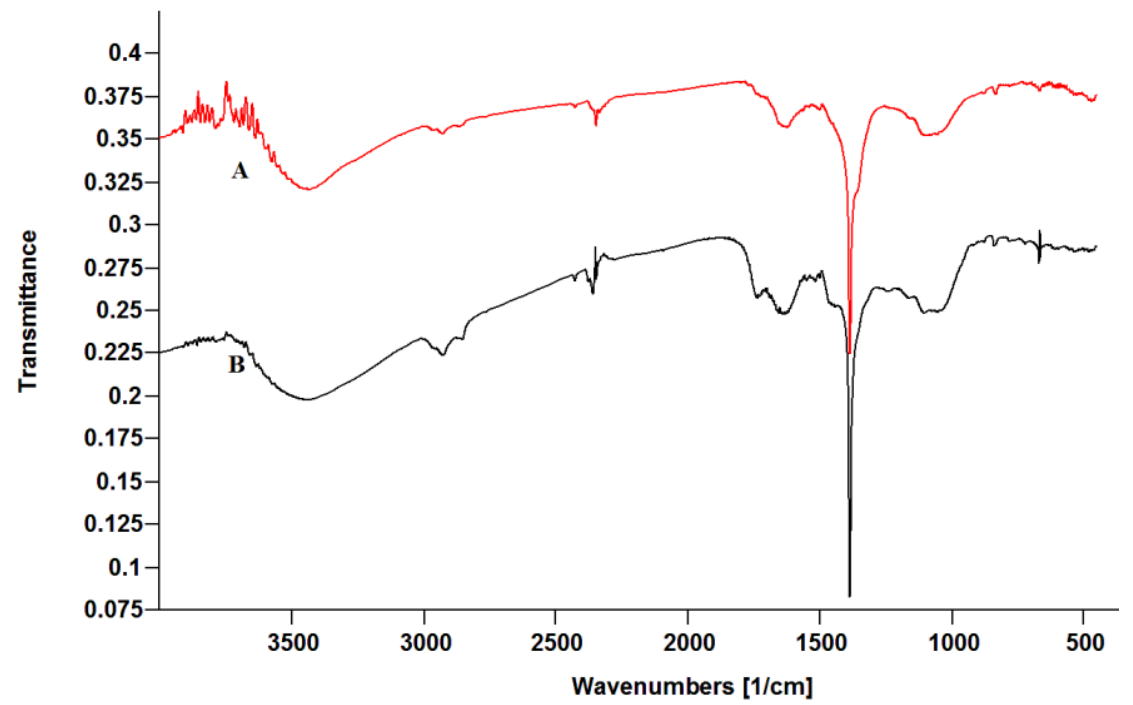

Fig. 5 FT-IR spectra of A) EC leaf powder and B) Synthesized AgNPs with capping of phytoconstituents

Catalytic activity of AgNPs on reduction of Methylene Blue: It is a well known fact that AgNPs and their composites show greater catalytic activity in the area of dye reduction and removal. Pure methylene blue has a max value of $664 \mathrm{~nm}$. Thirty minutes after the addition of the extract to the dye, the absorbance is gradually decreased and is shifted to higher wavelength. The decrease of absorbance is indicative of the ability of phytoextract to degrade methylene blue[13-15]. System containing dye, AgNPs and the extract at the end of 30 min time interval showed a marked decrease in the absorbance of methylene blue and increase of SPR peak of $\operatorname{AgNPs}$ (Fig. 6).

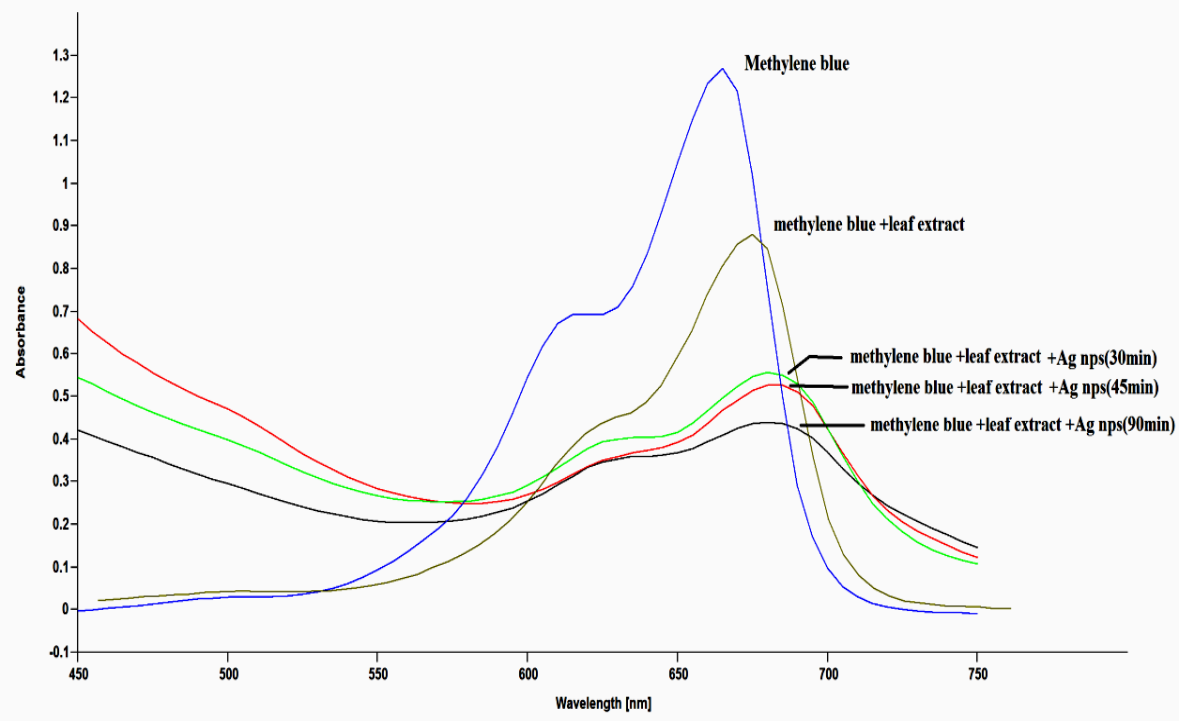

Fig.6 UV-visible spectra of methylene blue reduction by Eucalyptus leaves extract in the presence of AgNPs. 


\section{Conclusion}

We report a simple and efficient way to synthesis stable spherical AgNPs using eucalyptus leave extract. The synthesized AgNPs were initially confirmed by using UV-vis spectroscopy and TEM study. Crystalline nature of AgNPs was evidenced through XRD studies and multifunctional nature of plant extract was confirmed by FTIR studies. The synthesized AgNPs showed excellent catalytic activity in the reduction/degradation of methylene blue. Hence this synthesis process may be the best substitute of both chemical and other physical methods used in industry for large scale production of NPs.

\section{Acknowledgement}

The authors are thankful to UGC-DS Kothari Post Doctoral Fellowship programme for providing financial support.

\section{Reference}

[1] D. Sannino, V. Vaiano, O. Sacco and P. Ciambelli, "Mathematical modelling of photocatalytic degradation of methylene blue under visible light irradiation," Journal of Chemical Engineering, vol-1, pp.56-60, 2013

[2] B.S. Ratna and A. Padhi, "Pollution due to synthetic dyes toxicity \& carcinogenicity studies and remediation," International Journal of Environment Science, vol-3, pp.940-955,2012.

[3] L. Rastogi and J. Arunachalam, "Sunlight based irradiation strategy for rapid green synthesis of highly stable silver nano particles using aqueous garlic (Allium sativum) extract and their antibacterial potential," Materials Chemistry and Physics, vol-129, pp.558-563,2011.

[4] M.J. Ahmed, G. Murtaza, A. Mehmood and T.M. Bhatti, "Green synthesis of silver nanoparticles using leaves extract of Skimmialaureola: characterization and antibacterial activity," Material Letter, vol-153, pp.10-13,2012.

[5] B. Ajitha, Y.A.K. Reddy and P.S. Reddy, "Biosynthesis of silver nanoparticles using Momordi cacharantia leaf broth: evaluation of their innate antimicrobial and catalytic activities," Journal of Photochemistry and Photobiology B, vol-146, pp. 1-9,2015.

[6] N.R. Jana, Z.L. Wang and T. Pal, "Redox catalytic property of palladium nanoparticle: effect of electron donor acceptor and surfactant," Langmuir, vol-16,pp. $2457-2463,2000$.

[7] K. Mallick, M. Witcomb and M. Scurrell, "Silver nanoparticle catalysed redox reaction: an electron relay effect," Materials Chemistry and Physics, vol-97, pp.283-287,2006.

[8] Y. Guo and R.M. Bustin, "FTIR spectroscopy and reflectance of modern charcoals and

[9] fungal decayed woods: implications for studies of inertinite in coals," International Journal of Coal Geology,vol37,pp.29-53,1998.

[10] T-J. Ling, W-W. Ling, Y-J. Chen, X-C. Wan, T. Xia, X-F. Du, "Antiseptic activity and phenolic constituents of the aerial parts of Vitex negundo var. cannabifolia," Molecules, vol-15,pp.8469-77,2015.

[11] S. Mondal, N. Roy, R.A. Laskar, I. Sk, S. Basu, D. Mandal, "Biogenic synthesis of Ag, Au and bimetallic Au/Ag alloy nanoparticles using aqueous extract of mahogany (Swietenia mahogani JACQ.) leaves," Colloids and Surfaces B: Biointerfaces, vol-82,pp.497-504,2015.

[12] J.Y. Song, H.K. Jang and B.S. Kim, "Biological synthesis of gold nanoparticles using Magnolia kobus and Diopyros kaki leaf extracts," Process Biochemistry, vol-44,pp.1133-1138,2009.

[13] S. Kundu, S.K. Ghosh, M. Mandal and T. Pal, "Silver and gold nanocluster catalyzed reduction of methylene blue by arsine in micellar medium," Bulletin of Materials Science, vol-25,pp.577-9,2002.

[14] K. Mallick, M. Witcomb and M. Scurrell, "Silver nanoparticle catalysed redox reaction: an electron relay effect," Materials Chemistry and Physics, vol-97, pp.283-7,2006.

[15] N. Gupta, H.P. Singh and R.K.Sharma, "Metal nanoparticles with high catalytic activity in degradation of methyl orange: an electron relay effect," Journal of Molecular Catalysis A: Chemical , vol-335, pp.248-52,2011.

[16]H. Karimi, S. Mousavi, and B. Sadeghian, "Silver nanoparticle loaded on activated carbon as efficient adsorbent for removal of methyl orange," International Journal of Science Technology, vol5,pp.2346-2353,2012. 